\title{
Epitope Regions on U1 Small Nuclear RNA Recognized by Anti-U1RNA-specific Autoantibodies
}

\author{
R. M. Hoet, P. De Weerd, J. Klein Gunnewiek, I. Koornneef, and W. J. Van Venrooij \\ Department of Biochemistry, University of Nijmegen, $6500 \mathrm{HB}$ Nijmegen, The Netherlands
}

\begin{abstract}
Autoantibodies specifically directed to U1RNA were found in patients suffering from systemic lupus erythematosus (SLE) overlap syndromes. To obtain more insight in the mechanism responsible for this U1RNA-specific antibody formation and to use the antibodies eventually as a tool to study U1RNA-protein (U1RNP) interactions, the B cell epitopes on U1RNA were mapped. Using in vitro synthesized domains of U1RNA, the main epitope regions were found in stemloops II and IV. Furthermore, 3 '-end or 5 '-end truncation of both stemloop II and stemloop IV showed that the conformation of the stemloops is critical for antibody recognition. Mutant studies on both stemloops indicated that in the case of stemloop II the stem is the main antigenic region, whereas in stemloop IV, the loop (Eloop) is a main target. The results of this study support the idea that the anti-U1RNA autoantibody could be the result of a process driven by the human U1RNP complex itself (antigendriven process). (J. Clin. Invest. 1992. 90:1753-1762.) Key words: U1RNA • autoantibodies • autoantigens • autoimmunity - B cell epitopes
\end{abstract}

\section{Introduction}

Autoantibodies directed to components of RNA-protein particles (RNPs) ${ }^{1}$ are often found in sera from patients with a connective tissue disease (1). For instance, antibodies directed to (U)RNPs are found in systemic lupus erythematosus (SLE) and SLE overlap syndromes such as mixed connective tissue disease (MCTD) (2), antibodies directed to Ro and La RNPs are present in SLE and Sjögren's syndrome patients (3), antibodies directed to ribosomal proteins ( $\mathrm{P} 0, \mathrm{P} 1$, and $\mathrm{P} 2$ ) can be detected in patients with SLE (4) and the presence of antibody to transfer RNA (tRNA) synthetases are characteristic for the disease polymyositis (PM) (5). All of these RNPs play a major role in important cellular processes (for review see reference 1 ). Initially only antibodies directed to the protein part of these

Address correspondence to Dr. R. M. Hoet, Department of Biochemistry, University of Nijmegen, P.O. Box 9101,6500 HB Nijmegen, The Netherlands. 1992.

Received for publication 2 March 1992 and in revised form 1 June

1. Abbreviations used in this paper: IV-AS, antisense stemloop IV; MCTD, mixed connective tissue disease; PCR, polymerase chain reaction; PM, polymyositis; RNPs, RNA-protein particles.

J. Clin. Invest.

(C) The American Society for Clinical Investigation, Inc. $0021-9738 / 92 / 11 / 1753 / 10 \quad \$ 2.00$

Volume 90, November 1992, 1753-1762 particles were found $(1,2)$ but recently antibodies directed to the RNA moiety have been described as well. For example, antibodies directed to the GTPase activity center of $28 \mathrm{~S}$ rRNA have been found to coexist with anti-ribosomal $P$ protein antibodies (6) and, in PM patients, antibodies directed to an alanyl-tRNA synthetase as well as its cognate tRNA ${ }^{\text {Ala }}$ have been shown to occur (5). Also in patients who contain antibody directed to U1RNP, the presence of both anti-U1RNP protein and anti-U1RNA antibody has been demonstrated (7, 8). Surprisingly, antibodies directed to other (U)RNAs were not detected (8).

For several reasons it is important to know the precise regions on the U1RNA that are targeted by these antibodies. First it has been found that the antibody level to certain epitopes of U1RNA appear to correlate with disease activity and thus might play a role in the pathogenesis of the disease (9). A clear definition of such epitopes might be of diagnostic value. Second, knowledge of the epitope regions recognized by the antibodies might be useful in the study of RNA-protein interactions in these RNPs and might teach us more about the function of the U1RNA itself in the processing of pre-mRNA.

In this paper a detailed $B$ cell epitope mapping of human anti-U1RNA antibody is described. For this purpose a large number of patient sera as well as several mutants of U1RNA were used. It is shown that the main epitopes are located in the second and fourth stemloop structure and that the conformation of both stemloops is critical for antibody recognition.

\section{Methods}

Patients sera. Most of the patient sera were obtained from the Rheumatology Department of the University Hospital Nijmegen, the Netherlands. All patients used in this study obtained the diagnosis SLE overlap syndrome or MCTD according to criteria described previously (10). Sera from these patients all contain anti-(U)RNP antibody as detected by both immunoblotting and counterimmunoelectrophoresis. As a human control serum, a pool of 10 healthy blood donors was used.

Plasmids and DNA constructs. Full length human U1RNA (see Fig. $1 A$ ) was prepared by T7 transcription of the U1 DNA template (a kind gift of Dr. R. Spritz, Department of Medical Genetics, University of Wisconsin, Madison, WI [11]) linearized with MstII or HindIII. Template DNAs coding for the domains of U1RNA depicted in Fig. 1 $B$ were prepared as follows: stemloop I was prepared by $\mathrm{T} 7$ transcription of wild-type (wt) UIDNA linearized with MnI I (U1RNA position $47,3^{\prime}$ to stem I). Templates encoding stemloop II (U1RNA position 49-92), stemloop III (U1RNA position 93-118), and stemloop IV (U1RNA position 139-165) (see Fig. $5 A$ ) were all made by using oligonucleotides and polymerase chain reaction technology (all containing 5'EcoRI and 3'BamHI restriction sites) and ligated in the EcoRI and BamHI restriction sites of pGEM-3Zf $(+)$ (Promega Corp., Madison, WI). Domain IV-DE was prepared by digestion of Xenopus laevis wt U1DNA ( 12) with TaqI and BamHI (U1 position 117-165), mutant IV- $\triangle \mathrm{DE}$ by the same digestions of $X$. laevis $\triangle \mathrm{D}$ U1DNA (12) 
(U1RNA position 130-165), and mutant IV-D $\Delta E$ by the same digestions of $X$. laevis $\triangle E$ UIDNA (12) (U1RNA position 117-165, replacement of nucleotides $146-158$ by AGAA). DNA fragments IVDE, IV- $\Delta D E$, and IV-D $\Delta E$ were ligated in the ClaI and BamHI sites of pGEM-7Zf $(+)$ (Promega Corp.). DNA templates for stemloops II, III, and IV (E, DE, $\triangle D E$, and D $\triangle E$ ) of UIRNA were all linearized using BamHI digestion. Template DNA for preparing antisense stemloop IV (IV-AS) was made by EcoRI digestion of pGEM-3Zf $(+)$ containing stemloop IV. DNAs coding for U1RNA from common bean (Ulbean [13]), and for the mutants U1.4 (14) and U2.4 (14) were kind gifts of Dr. I. Mattaj (EMBL, Heidelberg, Germany). For transcription, Ulbean DNA (13) was linearized with SnaBI. Mutant U2.4, an U2RNA in which the B loop of U1RNA replaced the loop of stemloop IV of U2RNA (14) was digested with DraI. U1.4, a U1 mutant in which loop IV of U2RNA replaced the B loop of U1RNA (14) was digested with TaqI (cuts at position 117 of template U1DNA). Templates for the point mutants of the B loop of $\mathrm{Ul}$ (position 69 [A, C, U] and position $72[A, G]$ ) (kind gifts of $R$. Spritz [11]) were digested with TaqI before transcription. The B loop mutant B1 (for structure see Fig. $4 A$ ) and the E loop mutants (E1, E4, E8, E20, EJ) (see Fig. $5 A$ ) were all made by oligonucleotide-directed mutagenesis (Amersham, Amersham, UK) using the wt stemloop II and wt stemloop IV DNAs. For transcription they were linearized with BamHI. The correct identity of all point mutants and all individual stemloops was confirmed by DNA sequence analysis.

In vitro transcription and immunoprecipitation. For in vitro transcription, the T7 RNA polymerase system was used as previously described (8). The SP6 RNA polymerase system (Promega Corp) was used only for the production of antisense stemloop IV. The most stable secondary structures of RNAs made by in vitro transcription (point mutants and individual domains), were all predicted by the computer program $M$ fold (version 2) (15) using the U1RNA structure published by Krol et al. (16) as a reference. Additionally transcribed vector and linker sequences, present in some of the RNAs, had no influence on the predicted secondary structures.

All immunoprecipitations were performed at $500 \mathrm{mM} \mathrm{NaCl}, 0.05 \%$ NP-40, and $0.1 \%$ Tween-20 as previously described (8).

Alkali-digested RNA ladders. DNA templates for stemloop II and stemloop IV (see Fig. $3 A$ ) were transcribed using T7 RNA polymerase as described above. For the 5 '-end labeling, RNA was first dephosphorylated with calf intestinal alkaline phosphatase (Boehringer Mannheim, Germany) and then 5'-end-labeled with T4 polynucleotide kinase (Boehringer Mannheim GmBH, Mannheim, Germany) and [ $\gamma^{32}$ P]ATP (Amersham) (17). The RNA was 3'-end labeled by the terminal addition of $\left[{ }^{32} \mathrm{P}\right] \mathrm{pCp}$ (Amersham) using T4 RNA ligase (Pharmacia LKB, Piscataway, NJ) (17). The end-labeled RNAs were purified by gel electrophoresis before hydrolysis. Partial alkaline treatment using a solution of $33 \mathrm{mM}$ sodium bicarbonate at $90^{\circ} \mathrm{C}$ for $4 \mathrm{~min}$ was performed as described by Query et al. (18). Alkali digests were incubated with anti-U1RNA antibody coupled to protein A agarose under the conditions of the standard immunoprecipitation assay (8). The immunoprecipitated RNAs were separated on a urea-polyacrylamide gel. As a marker, end-labeled stemloop II and IV RNAs were partially digested with RNase $\mathrm{T} 1$ in $7 \mathrm{M}$ urea, $25 \mathrm{mM}$ sodium acetate ( $\mathrm{pH}=5.2), 1 \mathrm{mM}$ EDTA at $50^{\circ} \mathrm{C}$ and electrophoresed in parallel with the immunoprecipitated samples.

Competition assays. Competition assays were performed using the previously developed nitrocellulose binding assay (18a). 1 pmol ${ }^{32} \mathrm{P}$-labeled mutant RNA was mixed on ice with increasing amounts of unlabeled wt RNA (stemloop II or IV), $10 \mu \mathrm{g}$ yeast RNA in PBS containing $0.5 \mathrm{mM}$ 1,4-dithioerythritol (DTE). Subsequently serum $(0.1 \mu \mathrm{l})$ was added to all samples. After a 2-h incubation on ice, the immune complexes were collected on nitrocellulose and the radioactivity was determined. As a reference, 1 pmol of radiolabeled wt stemloop II or wt stemloop IV was incubated with increasing amounts of their unlabeled equivalent in the same assay to calculate the relative binding affinity of the mutants.

\section{Results}

Selection of patient sera specifically precipitating UIRNA. About 100 anti-U1RNP-positive patient sera were screened for the presence of anti-U1RNA antibody using a previously described immunoprecipitation assay in which ${ }^{32} \mathrm{P}$-labeled total HeLa cell RNA was the antigen source. The precipitations were carried out under stringent conditions ( $500 \mathrm{mM}$ salt [8]). As a result of this screening, 45 patients' sera were selected for their ability to precipitate UIRNA.

To ascertain that we were dealing with antibodies specifically directed to U1RNA, a number of filter-binding competition assays were performed. In vitro transcribed radiolabeled U1RNA was mixed with an increasing amount of competitor nucleic acid (dsDNA [PGEM-7Zf(+)], yeast RNA, Escherichia coli tRNA, poly A, poly $G$, poly $C$, poly [G-U], poly G-poly C, U2RNA, and U1RNA) and subsequently incubated with a patient serum containing anti-U1RNA antibody. After a 2 -h incubation on ice, immune complexes containing radiolabeled U1RNA were collected on nitrocellulose filters using a dot-blot manifold and the radioactivity on the filters was determined.

No competition was observed in case of poly $A$, poly $G$, poly C, poly (G-U), yeast RNA, dsDNA, $E$. coli tRNA, and U2RNA in concentrations exceeding a 1,000-fold molar excess of the competitor. In some sera a slightly diminished binding (up to $15 \%$ ) could be observed using poly G-poly C as competitor. However, increasing the poly $\mathrm{G}$-poly $\mathrm{C}$ concentration to a 10,000-fold molar excess did not decrease the binding of U1RNA to the filter any further, indicating that the anti-(poly G-poly C) antibodies do have another specificity than the antiU1RNA antibody. From these competition experiments it was concluded that the anti-RNA antibodies in the patient sera are predominantly U1RNA-specific.

To determine whether the anti-U1RNA antibodies detected in the patient sera were from the IgG or IgM class, biotinylated anti-human IgM (mu-chain) and biotinylated antihuman IgG (Fc-fragment) were used. Radiolabeled U1RNA and a patient serum were incubated for $2 \mathrm{~h}$ at $4^{\circ} \mathrm{C}$ followed by an incubation with biotinylated anti-IgG or biotinylated antiIgM bound to streptavidin-agarose. The labeled U1RNA indirectly bound to the streptavidin-agarose was then analyzed on a urea-polyacrylamide gel and the radioactivity was measured. In all 10 patient sera analyzed in this way, the ratio of antiU1RNA IgG versus IgM antibody was $>20$ (data not shown).

Epitope regions on UIRNA recognized by the autoantibodies. To characterize the main epitopes on U1RNA recognized by the patient antibodies, DNA templates coding for the individual domains of U1RNA were cloned behind the T7 RNA polymerase promoter (see Methods). These domains, depicted in Fig. $1 B$, constitute the stemloops I, II, III, and IV, the latter in three variants namely IV-DE, containing stemloop IV plus the single-stranded Sm binding site, IV-D $\Delta \mathrm{E}$ in which the top of stemloop IV was mutated, and IV- $\Delta \mathrm{DE}$ in which the singlestranded Sm binding site was deleted.

In vitro transcribed radiolabeled RNAs I, II, III, and IV-DE were mixed in equimolar amounts together with wt U1RNA as an internal control and used in immunoprecipitation assays at $500 \mathrm{mM} \mathrm{NaCl}$ with the 45 anti-U1RNA positive sera (see Methods). Some of the results of this type of experiment are shown in Fig. 2. 


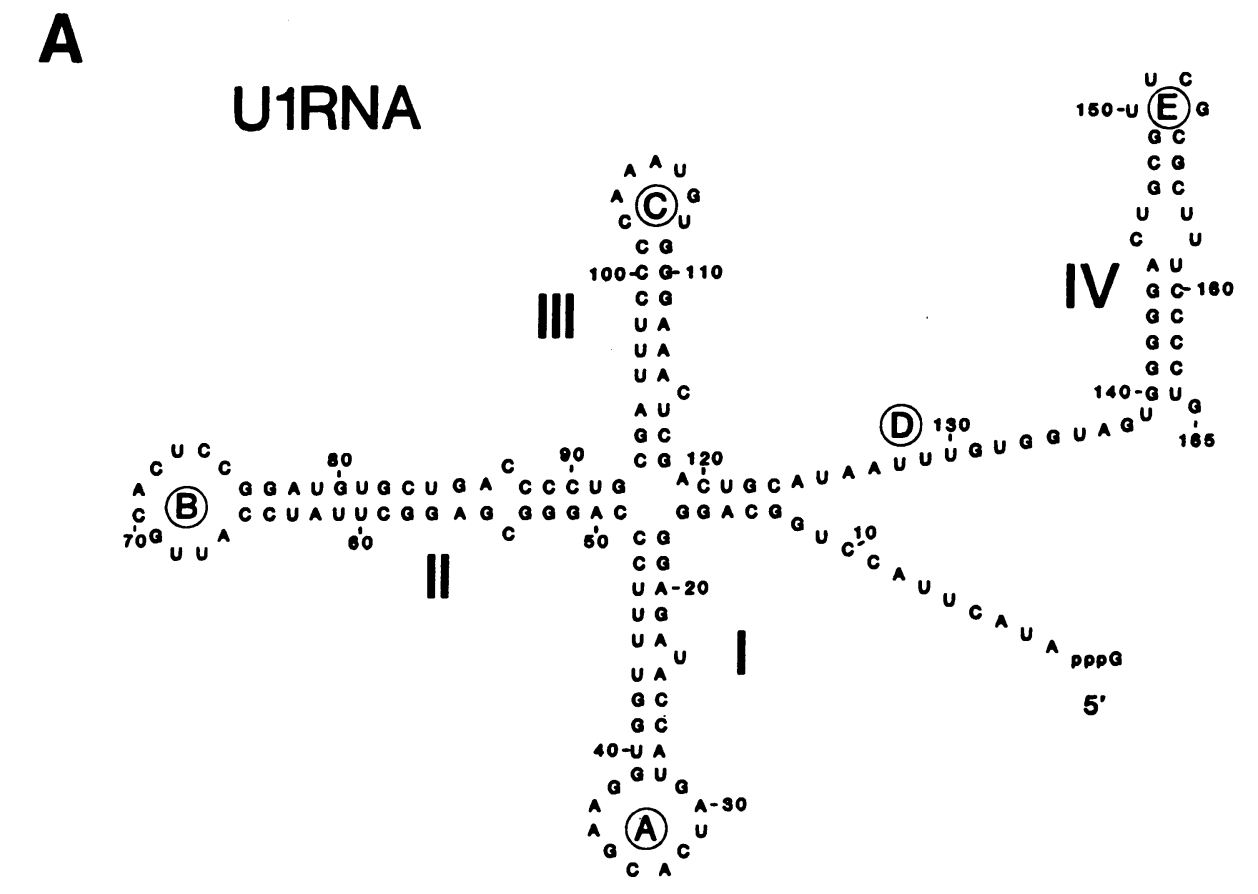

B
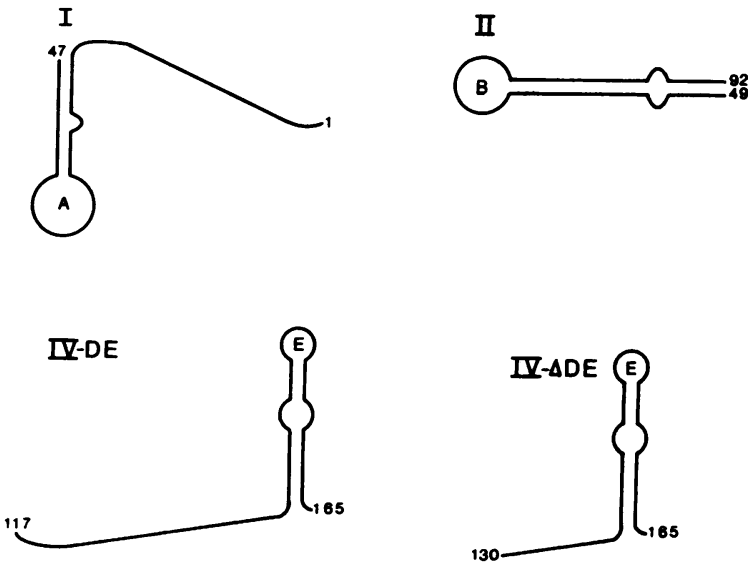

It is evident that most sera recognize stemloop II and domain IV-DE of U1RNA ( see Fig. $2 A$ ). To distinguish between antibodies directed to the single-stranded Sm-binding domain (D) or to stemloop IV, additional immunoprecipitations were performed with mutants IV-D $\Delta E$ and IV- $\Delta D E$ (for structures see Fig. $1 B$ ). The results clearly demonstrate that the autoantibodies recognize the stemloop IV structure (IV- $\triangle D E$ ) and not the Sm-binding domain contained in mutant IV-D $\Delta E$ (compare Figure $2 B$ and $C$ ). The overall results of this $B$ cell epitope mapping, summarized in Table I, show that 29 out of the 45 patient sera recognize both stemloop II and IV, while 8 sera recognized stemloop IV only and 2 sera reacted only with stemloop II. In six sera some weak reactivity directed to stemloop I and/or III could be detected as well (not shown in Fig. 2).
Figure 1. Secondary structures of UIRNA and UIRNA domains. $(A)$ Secondary structure of human U1RNA. The structure shown is as described by Krol et al. (16). Roman numerals I-IV indicate the various stemloops. Letters $A-E$ indicate single-stranded domains. $(B)$ Domains of U1RNA tested for antibody reactivity. Arabic numerals indicate the position of the nucleotides in U1RNA and correspond to arabic numerals in $A$. DNA templates used to synthesize these RNA domains were prepared as described in Methods. In most RNAs additional polylinker nucleotides were present, i.e., stemloop II; 5 '-end 10 nucleotides, 3 '-end 5 nucleotides, Stemloop III; 5'-end 10 nucleotides, 3 '-end 5 nucleotides, stemloop IVDE, IV- $\triangle D E$, and IV-D $\Delta E$; 5 '-end 71 nucleotides, 3 '-end 5 nucleotides. For details see Methods.
To investigate whether there was any cross-reactivity between activities recognizing stemloop II and IV, antibodies were affinity purified. First, protein A-selected immunoglobulins of anti-U1RNA positive sera were absorbed to biotin-labeled stemloop II or stemloop IV- $\triangle D E$ RNA. Then stemloop II or IV specific antibodies were selected using streptavidinagarose and subsequently eluted with $0.1 \mathrm{M}$ glycine $(\mathrm{pH}$ $=2.5$ ). In an immunoprecipitation assay using the radiolabeled second and fourth stemloop as antigen (similar to experiments shown in Fig. $2 \mathrm{~A}$ ), it then could be demonstrated in 10 patients' sera containing both reactivities that there was no cross-reactivity between antistemloop II and antistemloop IV antibodies. It is concluded that there are at least two antiUIRNA-specific antibody populations, one recognizing stem- 
$\mathbf{A}$

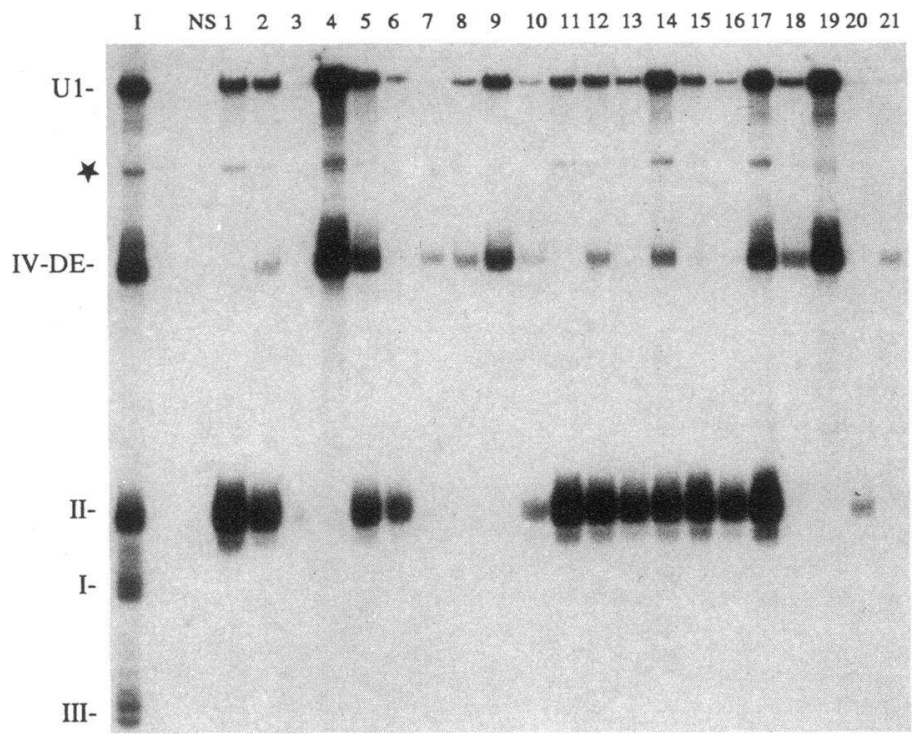

B

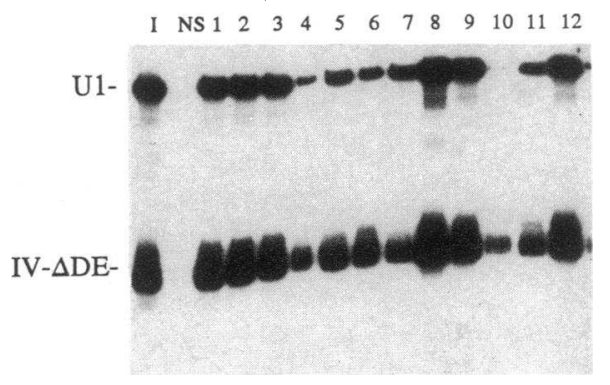

C I NS $1 \begin{array}{llllllllllllll}2 & 2 & 3 & 4 & 5 & 6 & 7 & 8 & 9 & 10 & 11 & 12\end{array}$

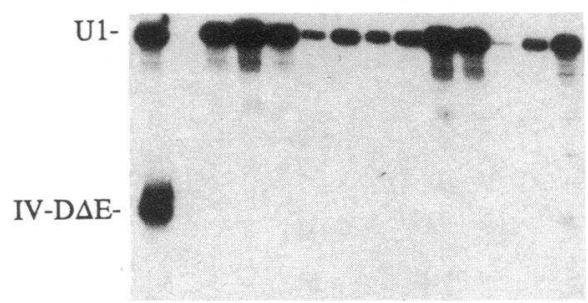

Figure 2. Immunoprecipitation of radiolabeled domains of U1RNA by patient antibodies. Immunoprecipitations were performed as described previously (8) and the immunoprecipitated RNAs (for structures see Fig. 1) were analyzed on $10 \%$ polyacrylamide-8.3 $\mathrm{M}$ urea gels. In $A, B$, and $C$, NS represents immunoprecipitation with a control normal serumpool. $(A)$ I, $10 \%$ of the input of the RNA domains (I, II, III, and IV-DE) and U1RNA. Lanes 1-21 represent immunoprecipitations with 21 anti-U1RNA positive patient sera. *, degradation product of U1RNA. (B) I, $10 \%$ of the input of IV- $\triangle D E$ RNA and UIRNA. Lanes $1-12$ correspond to immunoprecipitations with 12 patient sera containing antibody to domain IV-DE. $(C)$ I, 10\% of the input of IV-D $\triangle E$ RNA and UIRNA. Lanes 1-12 correspond to immunoprecipitations of the same 12 patient sera as used in $B$.

loop II and another one recognizing stemloop IV. Also competition experiments using stemloop II (radiolabeled) competed with wt stemloop IV (unlabeled) and vice versa (data not shown), indicated that there is virtually no cross-reaction between the two antibody populations.

The stemloop structure is necessary for antibody recognition. To obtain more insight in which part of the stemloops is important for binding of the antibodies, RNAs of stemloops II and IV were either 3 '-end labeled using [ $\left.{ }^{32} \mathrm{P}\right] \mathrm{pCp}$ and RNA ligase or 5'-end labeled using $\gamma^{32}$ PATP and T4 polynucleotide kinase and then hydrolyzed partially with sodium bicarbonate at $90^{\circ} \mathrm{C}$ (see Methods). Products still able to bind antibody were subsequently recovered by immunoprecipitation at 500 $\mathrm{mM}$ salt using protein A-agarose and analyzed on a polyacrylamide sequence gel (Fig. $3 B$ and $C$ ). As shown in Fig. $3 A$, the RNA of stemloop II is, at the $3^{\prime}$ end, extended by 36 nucleotides of the transcribed polylinker sequence. The immunoprecipitation experiments showed that the 36 extra nucleotides and 2 nucleotides of stem II could be removed without effect on antibody recognition (see Figure $3 \mathrm{~B}$, bottom; lanes 1-6). However, when additional " 3 ' nucleotides" were removed the RNA was not precipitable anymore. At the 5' end of stemloop II only 10 extra polylinker nucleotides were present. Also in this case few or zero nucleotides could be removed from stemloop II without effect on antibody recognition (Fig. $3 B$, lanes 1-6).

Similar results were obtained with stemloop IV. As shown in Fig. $3 A$, the RNA of stemloop IV- $\Delta \mathrm{DE}$ is, at the $5^{\prime}$ end, extended by 81 nucleotides ( 71 transcribed polylinker nucleotides plus a $\Delta \mathrm{D}$ sequence of 10 nucleotides). These 81 extra nucleotides appear not to be necessary for antibody recognition (see Fig. 3 C, top; lanes 1-6). Only in some cases (lanes 2 and 3 ) it was possible to remove up to 3 nucleotides from the $5^{\prime}$ end of stem IV without complete loss of antibody recognition. At the $3^{\prime}$ end of stemloop IV only five polylinker nucleotides are present and they could be removed without loss of antibody reactivity (Fig. $3 C$, bottom). Also in this case, $3^{\prime}$-end trimming of the hairpin abolished precipitation by the antibody (lanes 1-4).

These results thus indicate that in both the second and fourth stemloop the conformation of the stemloop structure appears to be necessary for recognition by the antibodies. This

Table I. Epitope Mapping of 45 Anti-U1RNA Positive Sera

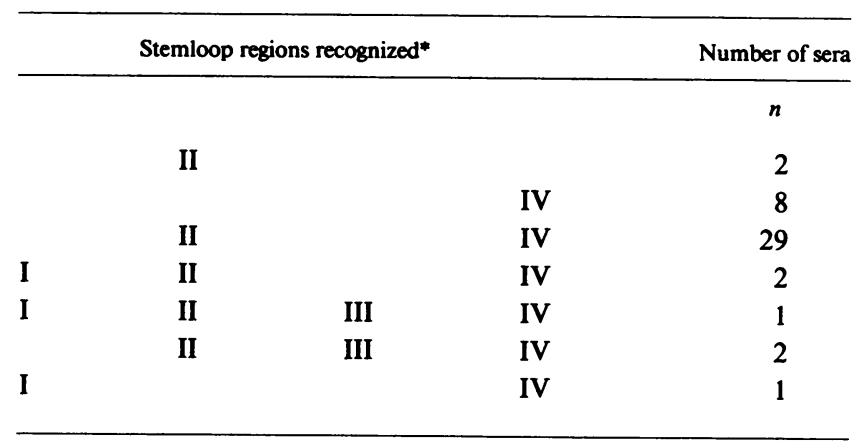

* Sera were considered monospecific for a particular stemloop if after long exposure of the autoradiogram only one signal, representing one stemloop region, could be detected. 
A

\section{Construct stemloop II Construct stemloop IV}

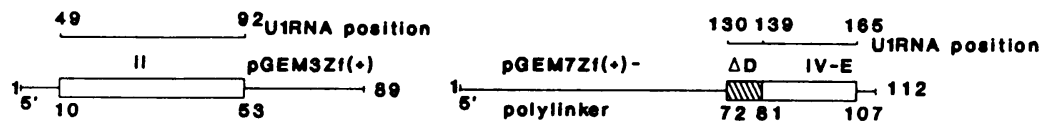

B

STEMLOOP II

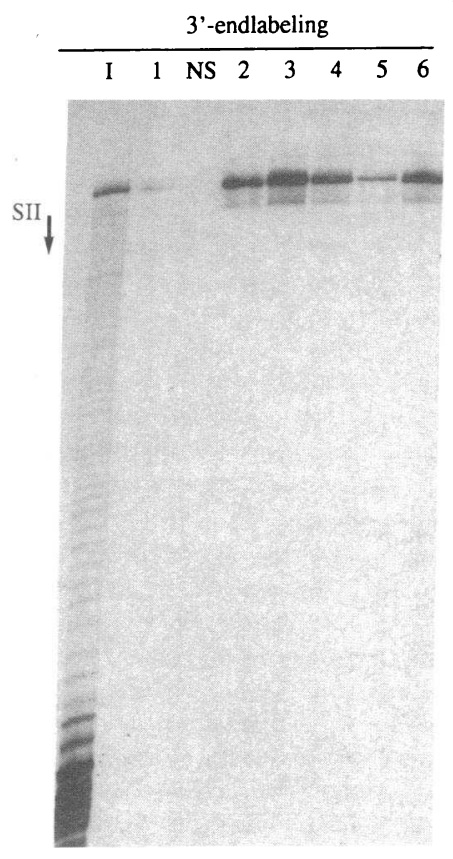

5'-endlabeling

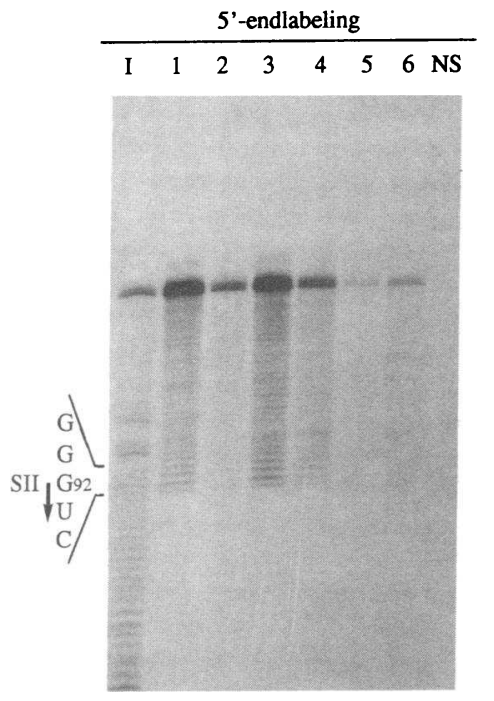

C

\section{STEMLOOP IV}

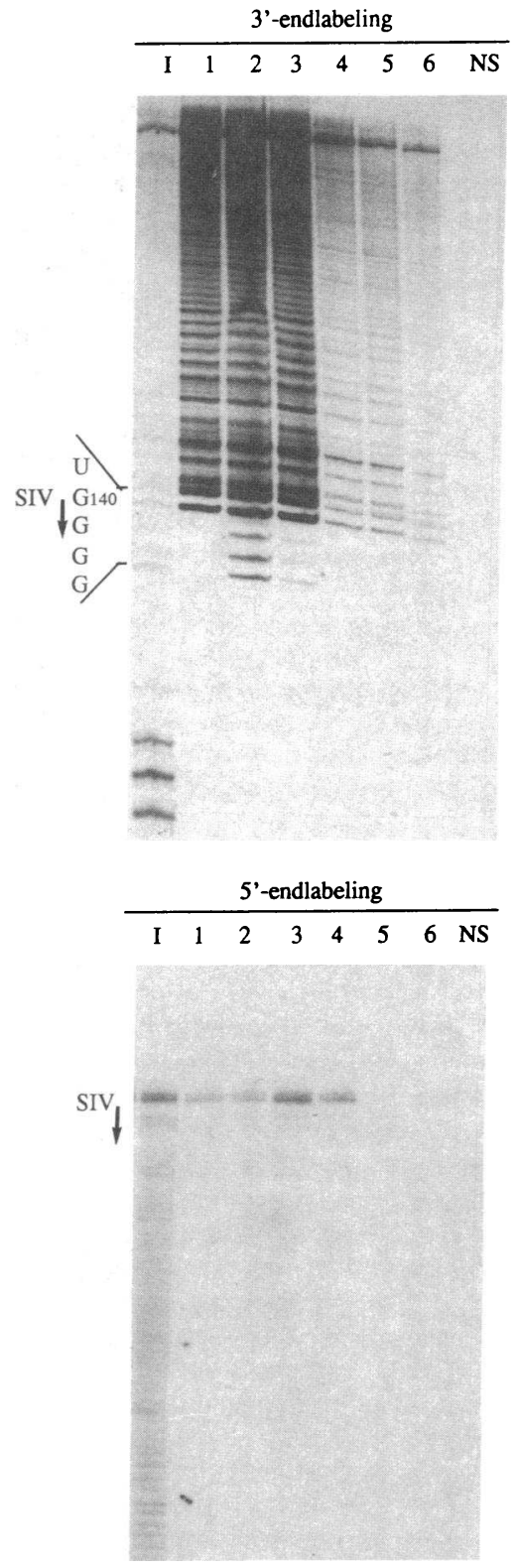

Figure 3. Boundaries of stemloop II and stemloop IV RNA required for interaction with their specific antibody. Stemloop II and IV RNAs $(A)$ were 3 '- and 5'end labeled, purified, partially hydrolyzed with alkali, and immunoprecipitated by patient antibodies under high salt conditions as described in Methods. Immunoprecipitated RNAs were analyzed on denaturating $15 \%$ polyacrylamide-8.3 $\mathrm{M}$ urea gels $(B$ and $C$ ). As a marker, 3'- and 5'-end-labeled stemloop II and IV RNAs, partially digested with RNase T1, were used (not shown). The arrows indicate the starting point of stemloop II (SII) and stemloop IV (SIV). (A) RNA constructs used for the alkaline hydrolysis. ( $B$ and $C$ ) Analysis of fragmented RNAs precipitated by patient antibodies. I, $10 \%$ of the input of the alkalihydrolyzed end-labeled RNAs. Lanes 1 and 2, precipitations with two patient sera monospecific for stemloop II $(B)$ or stemloop IV $(C)$. Lanes 3-6, precipitations with four patient sera recognizing both stemloops II and IV. NS, precipitations with a control normal serum pool. Note: ( $C$, bottom, lanes 5 and 6) In a longer exposure of the autoradiogram (not shown) weak but specific bands could be seen at the same height as in lanes 1-4. was the case for patient sera recognizing stemloop II only (Fig. $3 B$, lanes $l$ and 2 ), stemloop IV only (Fig. $3 C$, lanes 1 and 2 ), or recognizing both stemloops (Fig. $3 B$ and $C$, lanes 3-6).

The same type of experiment as shown in Fig. 3 for stemloops II and IV was also performed for stemloop I with serum B156. Also in this case it was found that only a few nucleotides could be removed from the stem of stemloop I without loss of antibody reactivity (data not shown).
The stem of stemloop II and the loop of stemloop IV are important antigenic determinants. To study the site of antibody recognition in more detail, several mutants of both stemloop II and stemloop IV as well as U1RNAs from other species (Figs. 4 $A$ and $5 A$ ) were made in vitro and tested in two types of assays. First, all mutants were tested in an immunoprecipitation assay under high salt conditions, using U1RNA or wt stemloop II or IV as an internal positive control. Results of these experiments 

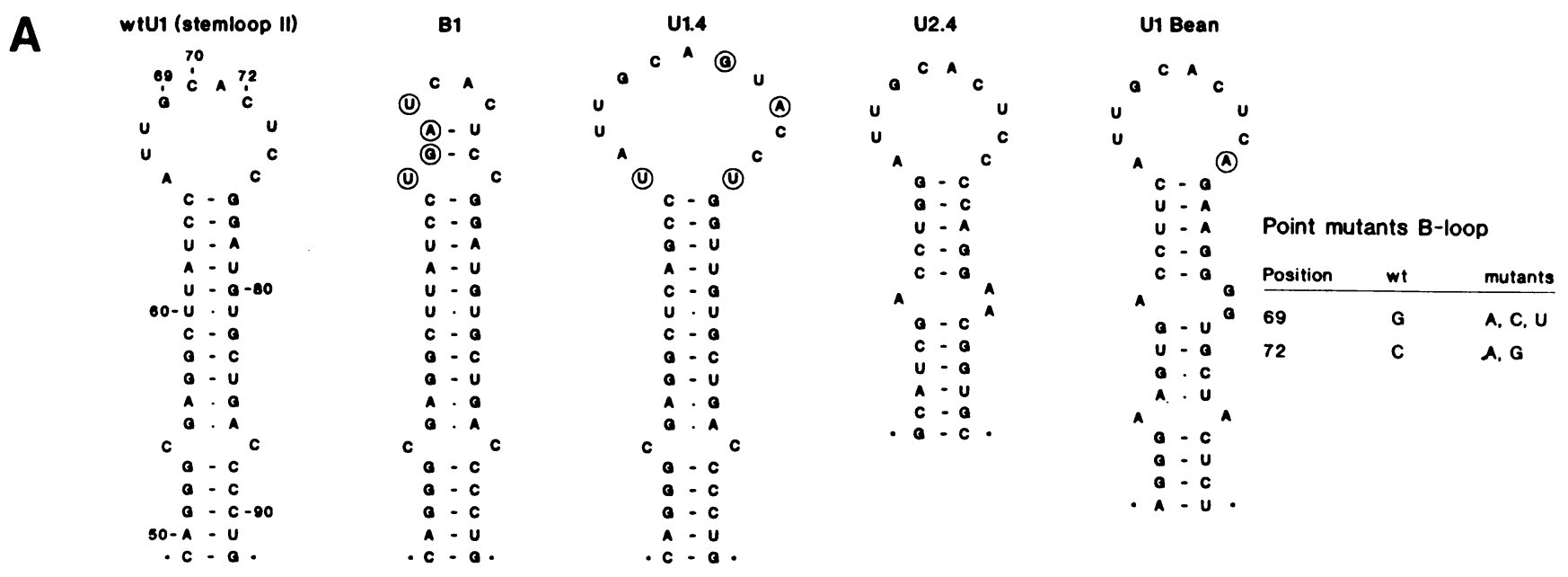

B
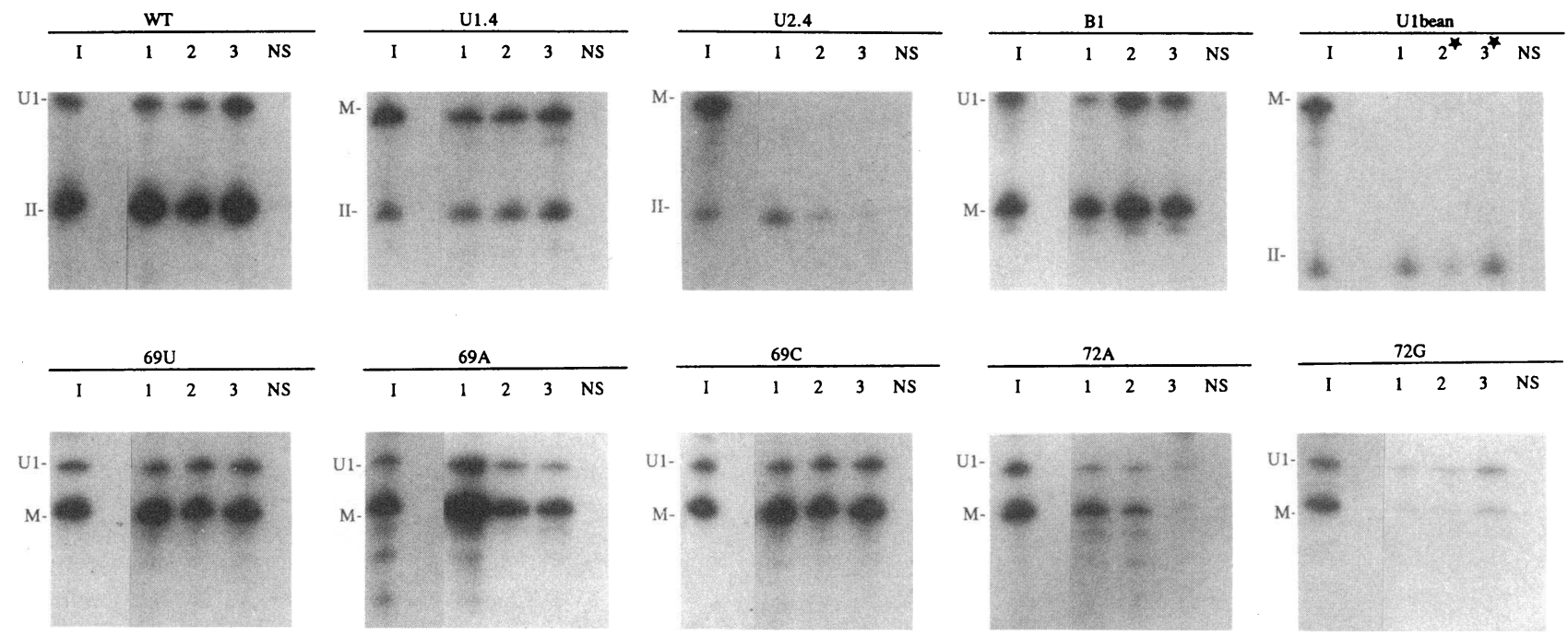

Figure 4. Binding of anti-U1RNA antibodies to stemloop II mutants of U1RNA. $(A)$ Predicted secondary structures for mutant and wt stemloop II RNA as calculated by the computer program M fold (15), using a previously described model for U1RNA (16). Encircled nucleotides represent mutations in the loop sequence compared with wt stemloop II. Some RNAs contained additional polylinker (p) or (U)RNA nucleotides. wtU1 (stemloop II) and B1; 5 '-end 10 (p)nucleotides, 3'-end 5 (p)nucleotides, U1.4 and point mutants B loop; 5 '-end nucleotides 1-48 of U1RNA, 3'-end nucleotides 93-117 of U1RNA, U2.4; 5'-end nucleotides 1-147 of U2RNA, 3'-end 5 (p)nucleotides, U1bean; full-length U1RNA (common bean) (nucleotide 1-163). For details see Methods. ( $B$ ) Immunoprecipitation of in vitro-transcribed radiolabeled mutants of stemloop II RNA analyzed on denaturing $10 \%$ polyacrylamide- $8.3 \mathrm{M}$ urea gels. In each precipitation the amount of mutant RNA (M) was at least five times the amount of internal control RNA (wt stemloop II or wt U1RNA). Lane I, 10\% of the input. Lane 1, precipitations with a stemloop II-specific serum (K1). Lanes 2 and 3, precipitations with two sera which recognize both stemloop II and IV (G23 and Z 5, respectively). The asterisks indicate that in the case of Ul bean, precipitations with patients sera monospecific for stemloop II are shown. (sera K1, S26, and P28 [not mentioned in Table I], respectively). NS, precipitation with a control normal serum pool.

are shown in Figs. 4 and 5. In the second assay all mutant RNAs were tested in a filter-binding competition assay, using radiolabeled mutant RNA and increasing concentrations of wt stemloop RNA as competitor. This second assay allows an estimation of the relative binding affinity of the various RNAs tested (Table II).

Using the immunoprecipitation assay each mutant was tested with six patient sera (two stemloop specific sera and four sera which recognize both stemloops) and a normal human control serum. In Fig. $4 B$ typical results are shown for stemloop II. Lane 1 shows the precipitations with a stemloop II-spe- cific serum and lanes 2 and 3 show precipitations with two sera that recognize both stemloop II and stemloop IV.

First it was tested whether the stem, the loop, or both structures are involved in antibody recognition. Mutant U2.4, which contains the stem IV of U2RNA and the second (B) loop of U1RNA (Fig. $4 A$ ) is not recognized by any of the patient antibodies (Fig. $4 \mathrm{~B}$ ), although this same mutant has been shown to effectively bind the U1RNA-associated A protein (U1-A) (14). This result indicates that the nucleotide sequence of stem II is important for antibody binding. This could be further substantiated by the inability of stemloop II-specific 


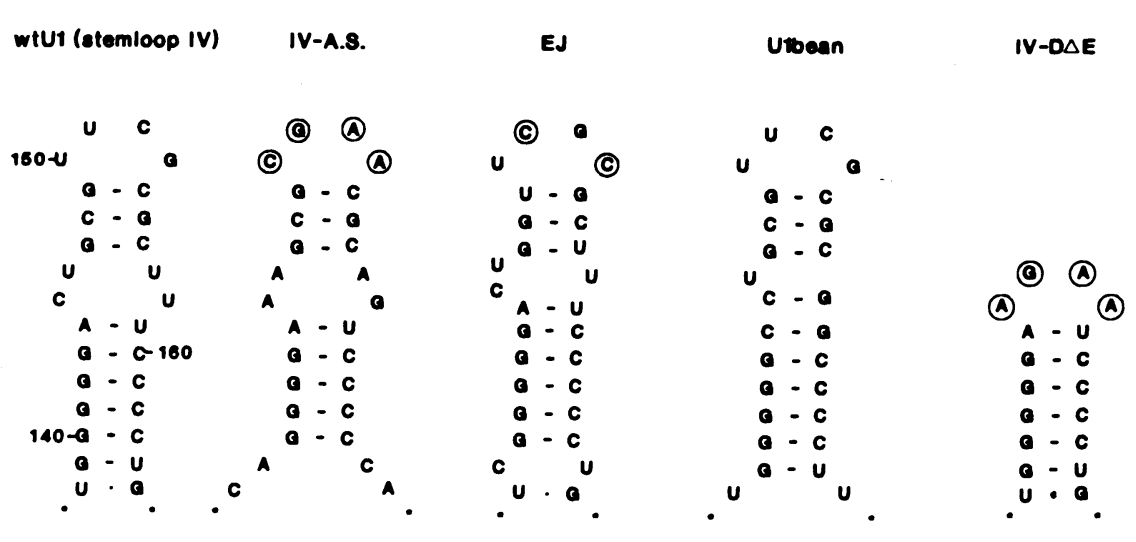

\begin{tabular}{|c|c|c|c|c|c|}
\hline \multicolumn{6}{|c|}{ Point mutants E-loop } \\
\hline Position & $w t$ & E1 & E4 & E8 & E20 \\
\hline 150 & $u$ & C & A & $A$ & C \\
\hline 161 & $u$ & C & A & C & C \\
\hline 162 & C & 0 & A & $\mathbf{u}$ & $\mathbf{u}$ \\
\hline 163 & $\mathbf{0}$ & C & $\mathbf{u}$ & C & C \\
\hline
\end{tabular}

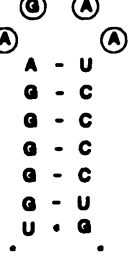

B
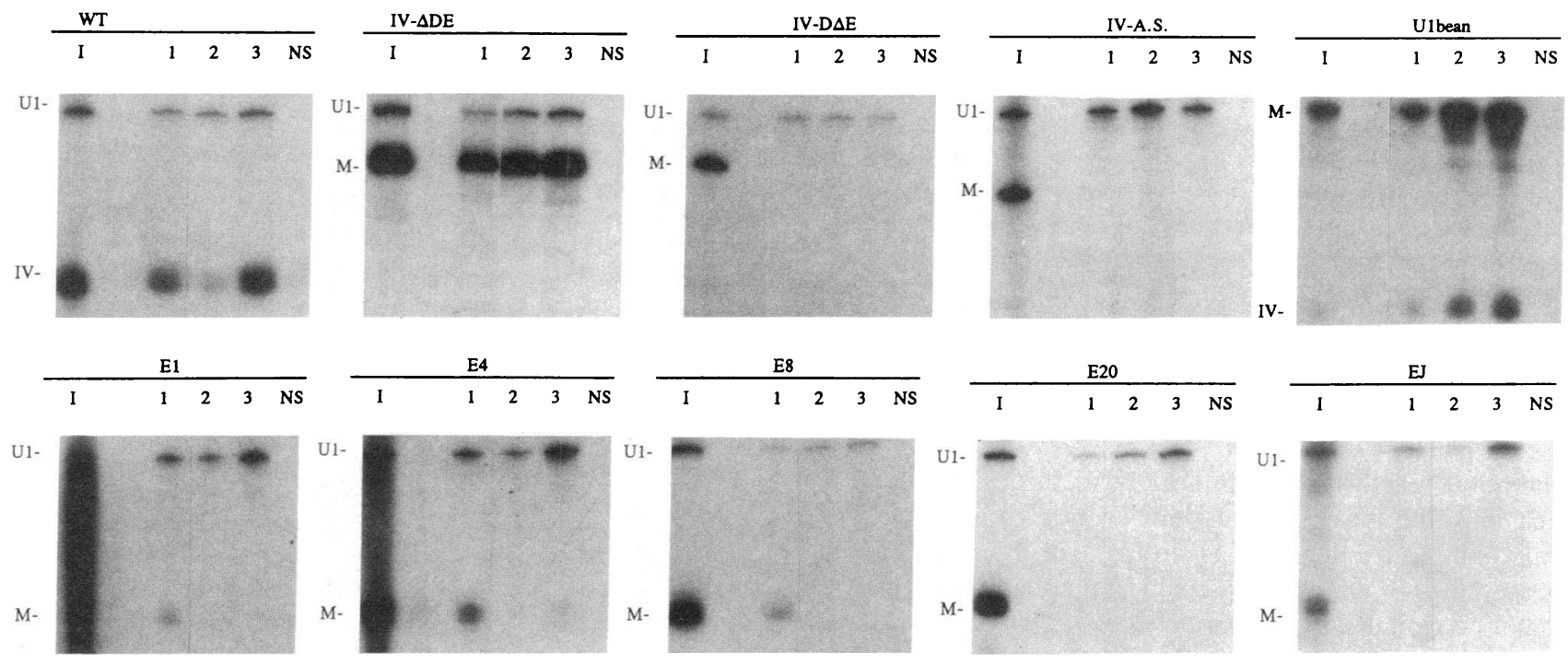

Figure 5. Binding of anti-U1RNA antibodies to stemloop IV mutants of UIRNA. ( $A$ ) Secondary structures of wt stemloop IV U1RNA as well as of several mutants of stemloop IV, predicted by the computer program $M$ fold (15) and the proposed model for U1RNA (16). Encircled nucleotides represent mutations in the loop sequences of the RNA. Most RNAs contained additional polylinker (p) or U1RNA nucleotides, i.e., wt U1 (stemloop IV) EJ and point mutants E loop; 5 '-end 10 (p)nucleotides, 3'-end 5 (p)nucleotides, IV-AS; 5 '-end 48 (p)nucleotides, 3 '-end 5 (p)nucleotides, U1bean; full-length UIRNA (common bean: nucleotide 1-163). For details see Methods. ( $B$ ) Immunoprecipitations of patients sera with in vitro-transcribed radiolabeled mutants of stemloop IV RNA analyzed on denaturing $10 \%$ polyacrylamide- $8.3 \mathrm{M}$ urea gels. In each precipitation the amount of a mutant RNA (M) was at least five times the amount of internal control (wt stemloop IV or wt U1RNA). Lane I, 10\% of the input. Lane 1, precipitations with a stemloop IV-specific serum (H13). Lanes 2 and 3, precipitations with two sera that recognize both stemloop II and stemloop IV (G23 and Z5, respectively). NS, precipitation with a control normal serum pool.

sera to precipitate Ulbean RNA (Fig. 4 B, lanes $1-3$ ) in which the stem II sequence is considerably different from that of human UIRNA whereas the loop sequence is almost identical ( 9 out of 10 nucleotides are identical, see Fig. $4 A$ ). It should be noted that in this case only sera containing stemloop II-specific antibody are shown because human antibodies that recognize stemloop IV do precipitate Ulbean RNA (Fig. $5 B$ ).

The reverse experiment, in which RNAs with an unchanged stem II and an altered loop B were used, showed that in contrast to the stem nucleotides, loop nucleotides are not essential for antibody recognition. For example, mutant U1.4 in which the second (B) loop of U1RNA is replaced by the fourth loop of U2RNA (see Fig. $4 A$ ) is well recognized by the antibodies (Fig. 4 B). This U1.4 RNA, however, has been shown not to bind the human U1-A protein (14). Also mutant $B 1$, in which four nucleotides of the sequence of the second (B) loop of U1RNA are changed (see Fig. $4 A$ ) is still able to bind the antibodies. In addition, point mutants in the B loop on position 69 and 72 do not abolish the binding of patient antibodies. From these experiments it is concluded that the stem II of UIRNA is an important site for antibody recognition.

Fig. $5 B$ shows the results obtained with antibodies directed to stemloop IV. In lane 1 a stemloop IV-specific serum was used and in lanes 2 and 3 two sera that recognize both stemloops were used.

All patient antibodies that recognize stemloop IV were 
Table II. Relative Binding Affinity of Patient Antibody for Stemloop II and Stemloop IV Mutants

\begin{tabular}{|c|c|c|}
\hline \multirow[b]{2}{*}{ RNA* } & \multicolumn{2}{|c|}{ Relative binding affinity } \\
\hline & Serum $\mathrm{ZS}^{*}$ & Serum G23* \\
\hline & \multicolumn{2}{|c|}{$\%$} \\
\hline Wt stemloop II & 100 & 100 \\
\hline B1 & 50 & 70 \\
\hline U1.4 & 40 & 30 \\
\hline U2.4 & $<1$ & $<1$ \\
\hline Ulbean & $<1^{8}$ & $<1^{8}$ \\
\hline 69A & 80 & 70 \\
\hline $69 \mathrm{C}$ & 10 & 20 \\
\hline $69 U$ & 80 & 70 \\
\hline $72 \mathrm{~A}$ & $<1$ & 10 \\
\hline $72 \mathrm{G}$ & $<1$ & 5 \\
\hline Wt stemloop IV & 100 & 100 \\
\hline IV- $\Delta \mathrm{DE}$ & 80 & 80 \\
\hline U1bean & 50 & 10 \\
\hline IV-D $\Delta \mathrm{E}$ & $<5$ & $<5$ \\
\hline IV-A.S. & $<1$ & $<5$ \\
\hline $\mathrm{E} 1, \mathrm{E} 4, \mathrm{E} 8, \mathrm{E} 20, \mathrm{EJ}$ & $<5$ & $<5$ \\
\hline
\end{tabular}

* Note: In all cases radiolabeled stemloop II or IV were used for the competition assays, except for U1bean and U2.4 where total radiolabeled (U)RNA was used (see Methods). " Sera Z5 and G23 contain antibodies directed to both stemloop II and IV. No competition with wt stemloop II was observed.

found not to react with mutant $\mathrm{D} \Delta \mathrm{E}$ ( structure see Fig. $5 A$ ) in which only the lower part of stem IV is present. Antisense stemloop IV RNA, in which, according to energy calculations, probably all G-C and A-U basepairs of the stem are present, is also not precipitated by any serum (Fig. $5 \mathrm{~B}$, IV-AS). Furthermore, Ulbean RNA, in which the central part of stem IV has an altered sequence but the upper part of the stem and the $\mathrm{E}$ loop are identical to the human sequence (Fig. $5 A$ ), is recognized very well by all antistemloop IV-positive sera (Fig. $5 B$ ). Taken together, these results indicate that sequences in the upper part of stem IV and in the E loop are important for antibody binding.

To test this in more detail, several E loop mutants (E1, E4, E8, E20, EJ ) were prepared and tested in the immunoprecipitation assay and in affinity-binding studies. The results indicated that among the serum antibodies at least two specificities could be discerned. One of them did not recognize any altered $E$ loop (Fig. $5 \mathrm{~B}$; E1, E4, E8, E20, EJ, lanes 2 and 3) while the second did precipitate mutants E1, E4, and E8 but in lower amounts compared with the precipitation of wt stemloop IV (Fig. $5 B$, lane 1). Coincidently, the patients sera containing the former specificity also contained antibody to stemloop II whereas two of the three patients sera containing the second idiotype were monospecific for stemloop IV.

The antibody-binding affinity of all these mutants was measured also quantitatively using two patient sera (Z5 and G23) that were tested earlier in the immunoprecipitation experiments (Figs. $4 B$ and $5 B$, lanes 2 and 3 ). In the quantitative filter-binding assay, equal amounts $(1 \mathrm{pmol})$ of radiolabeled mutant RNA and increasing concentrations of wt stemloop RNA as a competitor were used (see Methods). The relative binding affinity of a mutant RNA, as compared with wt stemloop RNA, was calculated as the concentration unlabeled wt RNA needed to obtain a $50 \%$ reduction of the radioactive signal divided by the concentration unlabeled wt RNA needed to obtain $50 \%$ reduction of the labeled wt RNA signal itself. The results tabulated in Table II support the conclusions drawn from the precipitation experiments, although it can be seen that all mutations affect the antibody binding affinity of the RNA. It is also clear that all point mutants of the E loop decrease the binding of antistemloop IV antibody considerably.

\section{Discussion}

Autoantibodies directed to U1RNA have been shown to be frequently present in serum from patients with SLE or SLE overlap syndromes (8). The finding that changes in the antibody levels directed to certain epitopes of U1RNA seem to correlate with changes in disease activity (9) suggest an involvement of these autoantibodies in the disease and underscores the importance of more detailed knowledge of the RNA structures targeted by these antibodies. Therefore the B cell epitope regions of UIRNA were mapped using a large number of patient sera and several structural domains and mutants of U1RNA (see Figs. $1 B, 4 A$, and $5 A$ ). The results showed that the main epitope regions are located in stemloops II and IV (Table I), a finding that is in agreement with the results of a previous study in which deletion mutants of U1RNA were used (8). Furthermore, immunoprecipitation experiments using 3'- or 5'-endlabeled RNA of stemloop II or IV, randomly hydrolyzed by alkali, strongly suggested that the conformation of both stemloop structures is critical for antibody recognition.

Further experiments were then performed to reveal whether, apart from the stemloop structure, a certain nucleotide sequence in the stem or the loop was important. The studies on stemloop II indicate that the main antigenic determinant is located in the stem structure. First, when the sequence and size of the loop were changed, as in mutants U1.4 or B1, the antibodies still were able to bind. Second, when the stem of stemloop II was changed in sequence and length, leaving the B loop unchanged, as in mutants U2.4 or U1bean, the anti-RNA antibody did not recognize these RNAs anymore. It is known that the U1RNA-associated A protein (U1-A), which recognizes the $B$ loop sequence AUUGCACXXX ( $X=$ random nucleotide) in the wt U1RNA $(14,19)$, binds efficiently to Ulbean and U2.4 RNA, indicating that the tertiary structure of the B loop in these RNAs is similar to that of wt U1RNA. On the other hand, U1-A does not associate with the B loop mutants U1.4 and B1 (U1.4 [14]; B1,.Dr. W. Boelens, unpublished results). Our conclusion therefore is that the binding sites of the U1-A protein and the antistemloop II antibody are different, implying an antiidiotype mechanism to explain the presence of antistemloop II antibody in these patients $(7,20)$ is unlikely. Furthermore, immunizations of mice with affinitypurified human anti-U1-A autoantibodies, recognizing the U1RNA-binding domain of U1-A $(14,19)$, did result in the production of antiidiotypic U1-A antibody, which, however, showed no detectable anti-U1RNA activity (our unpublished results). 
Although the main binding site of the antistemloop II antibody thus is located in the stem structure, it remains possible that some nucleotides in the loop sequence are important as well. For example, a point mutation at position 72 (in particular the mutation into a $G$ ) was reproducibly found to result in a much lower binding efficiency of the antibody, both in immunoprecipitation and in competition studies. Since replacement of the entire loop by another loop structure has only little effect on antibody binding, the mutation of position 72 probably affects the tertiary structure of the (stem)loop. Our conclusion that the stem of stemloop II is the major determinant for antibody recognition complements a previous study of Deutscher and Keene (7) who found, using two stemloop II specific antiU1RNA sera, that the loop sequence itself is not sufficient for antibody recognition.

In the case of stemloop IV the results are somewhat different. Again, correct folding of the stemloop structure is necessary for antibody recognition, but now the upper part of the stem and the $\mathrm{E}$ loop are the main regions targeted by the antibodies. This region of stemloop IV is very conserved in evolution, since it can be found unchanged in, for example, bean U1RNA. The E loop sequence as such can be found in several unrelated RNAs, for example, $E$. coli rRNAs (21). The tertiary structure of the very stable hairpin of stemloop IV, recently solved by NMR (22), may have a function in organizing the proper folding of complex RNA structures (21). The sequence of the E loop itself seems to be critical for antibody binding, because mutations resulted in a decreased binding of the RNA to the antibodies (see Fig. 5 and Table II). Because a number of mutants of the E loop (mutant E1, E4, E8) are still, though inefficiently, recognized by the antibodies in some, but not all sera, one may assume that there are at least two antibody specificities directed to stemloop IV.

It was discussed earlier that an antiidiotype mechanism is unlikely to be responsible for the production of antistemloop II antibody. In the case of antistemloop IV antibody, an antiidiotype mechanism seems unlikely as well because no antigenic protein is known to be associated with this part of the UIRNA. A more probable mechanism could be that the autoantibodies arise as a result of a primary immune response to a foreign antigen that shares epitopes with host macromolecules (molecular mimicry).

An alternative view is that the target autoantigen itself initiates and sustains autoantibody synthesis (antigen-driven mechanism). In the case of anti-U1RNA autoantibodies a crossreaction with a foreign antigen cannot be excluded. Although in the case of stemloop II we were unable to find such a "stem II-like" structure in the EMBL nucleic acid database (release 29.0), our results have shown that it is probably not only the nucleotide sequence but also the tertiary structure that is important for antibody recognition. Furthermore, the sequence of the E loop of stemloop IV has been found in several other RNAs as, for example, in $E$. coli rRNAs (21). On the other hand, the finding that in most sera more than one epitope on U1RNA is recognized, speaks in favor of an antigen-driven mechanism. The fact that anti-U1RNA antibodies always seem to coexist with autoantibodies directed to several U1RNP-proteins also supports this view. Moreover, affinitypurified anti-U1RNA antibody against stemloop II or IV does react exclusively with native U1RNPs, even when a total HeLa cell extract is used in the immunoprecipitation assay (Hoet et al., manuscript in preparation). This implies that at least in some native U1RNP complexes stem II and stemloop IV are free to interact with the antibody. These findings are compatible with the view that anti-U1RNA antibodies directed to these parts of the RNA might be the result of a process driven by the human U1RNP complex as the antigen.

Recent results obtained with another RNA antigen-antibody system also support the antigen-driven mechanism. Anti28S rRNA antibodies, present in sera from patients that also contain anti-P ribosomal protein antibody appear to recognize an epitope region in the $60 \mathrm{~S}$ ribosomal subunit, which lies close to but is probably not identical to the binding site of the $P$ proteins (23). The production of anti-28S rRNA antibody and anti-P protein antibody in the same patient thus seems to be related to the close proximity of these two antigens in the $60 \mathrm{~S}$ subunit rather than the result of an idiotype/antiidiotype mechanism (6). In conclusion, our results support the hypothesis that anti-U1RNA autoantibodies are produced via an antigendriven process. However, a primary event involving the crossreaction of antibodies with host epitopes, which may lead to tissue damage with the release of host antigens that fuel the autoimmune response further, still remains possible.

\section{Acknowledgments}

The authors are grateful to Dr. Iain Mattaj and Dr. Richard Spritz for supplying DNA templates of several U1RNA mutants. We thank Dr. Celia van Gelder (Department of Biochemistry, University of Nijmegen, The Netherlands) for assistence in secondary RNA structure predictions and Dr. Wilbert Boelens and Dr. Ger Pruijn (Department of Biochemistry, University of Nijmegen ) for helpful discussions and critical reading of the manuscript. Patients sera were kindly obtained from Dr. D. J. de Rooij and Dr. L. B. van de Putte via the Rheumatology Department of the University Hospital Nijmegen and St. Maartenskliniek, Nijmegen, The Netherlands.

These investigations were supported by the Netherlands Foundation for Chemical Research (SON) with financial aid from the Netherlands Organization for Scientific Research (NWO) and from the Technology Foundation (STW).

\section{References}

1. Tan, E. M. 1989. Antinuclear antibodies: diagnostic markers for autoimmune diseases. Adv. Immunol. 44:93-152.

2. van Venrooij, W. J., and P. T. G. Sillekens. 1989. Small nuclear RNA associated proteins, autoantigens in connective tissue diseases. Clin. Exp. Rheumatol. 7:635-645.

3. Slobbe, R. L., G. J. M. Pruijn, and W. J. van Venrooij. 1992. Ro(SS-A) and La (SS-B) ribonucleoprotein complexes: structure, function and antigenicity. Ann. Med. Interne. 142:592-600.

4. Elkon, K., S. Skelly, A. P. Parnassa, W. Moller, W. Danho, H. Weissbach, and N. Brot. 1986. Identification and chemical synthesis of a ribosomal P protein antigenic determinant in systemic lupus erythematosus. Proc. Natl. Acad. Sci. USA. 83:7419-7423.

5. Bunn, C. C., and M. B. Mathews. 1987. Autoreactive epitopes defined as the anticodon region of alanine transfer RNA. Science (Wash. DC). 238:11161119.

6. Chu, J.-L., N. Brot, H. Weissbach, and K. Elkon. 1991. Lupus antiribosomal $P$ antisera contain antibodies to a small fragment of $28 S$ rRNA located in the proposed ribosomal GTPase center. J. Exp. Med. 174:507-514.

7. Deutscher, S. L., and J. D. Keene. 1988. A sequence-specific conformational epitope on UI RNA is recognized by a unique autoantibody. Proc. Natl. Acad. Sci. USA. 85:3299-3304.

8. van Venrooij, W. J., R. Hoet, J. Castrop, B. Hageman, I. W. Mattaj, and L. B. van de Putte. 1990. Anti-(U1) small nuclear RNA antibodies in anti-small nuclear ribonucleoprotein sera from patients with connective tissue diseases. $J$. Clin. Invest. 86:2154-2160.

9. Hoet, R. M., I. Koornneef, D. J. de Rooij, L. B. van de Putte, and W. J. van 
Venrooij. 1991. Correlation between anti-U1RNA antibodies and disease activity. Mol. Biol. Rep. 15:193. (Abstr.)

10. de Rooij, D. J., L. B. van de Putte, W. J. Habets, A. L. Verbeek, and W. J. van Venrooij. 1988. The use of immunoblotting to detect antibodies to nuclear and cytoplasmic antigens. Scand. J. Rheumatol. 17:353-364.

11. Surowy, C. S., V. L. van Santen, S. M. Scheib-Wixted, and R. A. Spritz. 1989. Direct, sequence-specific binding of the human U1-70K ribonucleoprotein antigen protein to loop I of U1 small nuclear RNA. Mol. Cell. Biol. 9:4179-4186.

12. Hamm, J., M. Kazmaier, and I. W. Mattaj. 1987. In vitro assembly of U1 snRNPs. EMBO (Eur. Mol. Biol. Organ.) J. 6:3479-3485.

13. Hamm, J., V. L. van Santen, R. A. Spritz, and I. W. Mattaj. 1988. Loop I of U1 small nuclear RNA is the only essential RNA sequence for binding of specific U1 small nuclear ribonucleoprotein particle proteins. Mol. Cell Biol. 8:4787-4791.

14. Scherly, D., W. Boelens, N. A. Dathan, W. J. van Venrooij, and I. W. Mattaj. 1990. Major determinants of the specificity of interaction between small nuclear ribonucleoproteins U1A and U2B and their cognate RNAs. Nature (Lond.). 345:502-506.

15. Jaeger, J. A., D. H. Turner, and M. Zuker. 1989. Predicting optimal and suboptimal secondary structure for RNA. Methods Enzymol. 183:281-306.

16. Krol, A., E. Westhof, M. Bach, R. Lührmann, J.-P. Ebel, and P. Carbon. 1990. Solution structure of human U1 snRNA. Derivation of a possible three-dimensional model. Nucleic Acids Res. 18:3803-3811.
17. Sambrook, J., E. F. Fritsch, and T. Maniatis. 1989. Molecular Cloning: A Laboratory Manual. 2nd ed. Cold Spring Harbor Press, Cold Spring Harbor, NY.

18. Query, C. C., R. C. Bentley, and J. D. Keene. 1989. A specific 31-nucleotide domain of U1 RNA directly interacts with the 70K small ribonucleoprotein component. Mol. Cell. Biol. 9:4872-4881.

18a.Hoet, R. M., I. Kournneef, D. J. de Rooij, L. B. van de Putte, and W. J. van Venrooij. 1992. Changes in anti-UIRNA antibody levels correlate with disease activity in patients with SLE overlap syndrome. Arthritis Rheum. In press.

19. Tsai, R. E., D. S. Harper, and J. D. Keene. 1991. U1-snRNP-A protein selects a ten nucleotide consensus sequence from a degenerate RNA pool presented in various structural contexts. Nucleic Acids Res. 19:4931-4936.

21. Tuerk, C., P. Gauss, C. Thermes, D. R. Groebe, M. Gayle, N. Guild, G. Stormo, Y. D'aubenton-Carafa, O. C. Uhlenbeck, I. Tinoco, Jr., et al. 1988. CUUCGG hairpins: extraordinarily stable RNA secondary structures associated with various biochemical processes. Proc. Natl. Acad. Sci. USA. 85:1364-1368.

22. Cheong, C., G. Varani, and I. Tinoco, Jr. 1990. Solution structure of an unusually stable RNA hairpin 5'GGAC(UUCG)GUCC. Nature (Lond.). 346:680-682.

23. Uchiumi, T., R. R. Traut, K. Elkon, and R. Kominami. 1991. A human autoantibody specific for a unique conserved region of $28 \mathrm{~S}$ ribosomal RNA inhibits the interaction of elongation factors $1 \alpha$ and 2 with ribosomes. J. Biol. Chem. 266:2054-2062. 